\title{
Textile Antenna for First-Person View Goggles
}

\author{
Luis Andre ${ }^{1,2, *}$, Pedro Pinho ${ }^{1,2}$, Carolina Gouveia ${ }^{1,3}$, Caroline Loss ${ }^{4}$ \\ ${ }^{1}$ Instituto de Telecomunicacoes, \\ Aveiro, Portugal \\ ${ }^{2}$ Instituto Superior de Engenharia de Lisboa, \\ Lisboa, Portugal \\ ${ }^{3}$ Universidade de Aveiro, \\ Aveiro, Portugal \\ ${ }^{4}$ FibEnTech Research Unit, \\ Covilha, Portugal \\ a44681@alunos.isel.pt
}

\begin{abstract}
Unmanned Aerial Vehicles, also known as drones, are vehicles controlled remotely. First-Person View (FPV) technology allows these vehicles to have greater versatility and be more easily piloted. The antennas currently used for video transmission are bulky and uncomfortable, so there is a need for this volume to be reduced to increase portability, being this the focus of the paper. The antenna was developed in textile materials to reduce its size and weight, as these materials allow for a better comfort and a reduced footprint. This paper presents a microstrip array adapted for the frequencies used in FPV video transmission. The designed textile antenna was compared with a commercially available rigid antenna used for this transmission. The comparison shows promising results for the antenna made of textile materials. The main goal of increasing the comfort and portability of the FPV goggles was achieved.
\end{abstract}

Index Terms-Antennas; Array antennas; Drones; FPV; Textile Antenna.

\section{INTRODUCTION}

Unmanned Aerial Vehicles (UAVs), also known as drones, are aerial vehicles that can be controlled remotely and do not require a crew on board. With the increasing demand and interest in drones, the First-Person View (FPV) technology appears. FPV is a method used to pilot drones that allows the pilot to get a perspective as if he was inside the aircraft, thus making it possible to obtain a better control and a more useful perspective for those who fly the aircraft. This technology opens possibilities in several areas, such as intervention and rescue in dangerous environments [1]. In addition, in agriculture, e.g., allowing the farmer to observe agricultural fields more efficiently [2]. This technology can also be used in addition to virtual reality, for tourism marketing, exploring different places around the world in real-time [3]. Despite the number of applications, nowadays, the most explored fields on FPV are surveillance and Drone Racing competitions [4].

Manuscript received 1 February, 2021; accepted 6 April, 2021.

This work is funded by FCT/MCTES through national funds and when applicable co-funded EU funds under Grant No. UIDB/50008/2020UIDP/50008/2020 and Grant No. UIDB/00195/2020. This work is also funded by the Fundação para a Ciência e Tecnologia (FCT) through Fundo Social Europeu (FSE) and by Programa Operacional Regional do Centro under the PhD Grant No. SFRH/BD/139847/2018.
To have a functional FPV system, three components are needed:

1. A drone, which is the aircraft that will be piloted;

2. A controller that allows the pilot to control the drone;

3. The FPV goggles that allow the pilot to view what is captured by the drone's camera, as shown in Fig. 1.

Observing Fig. 1, it is also possible to see that the FPV goggles have two different receiver antennas, an omnidirectional and a directional one, to ensure that the video reception will be continuous independent of the drone's position (transmitter antenna).

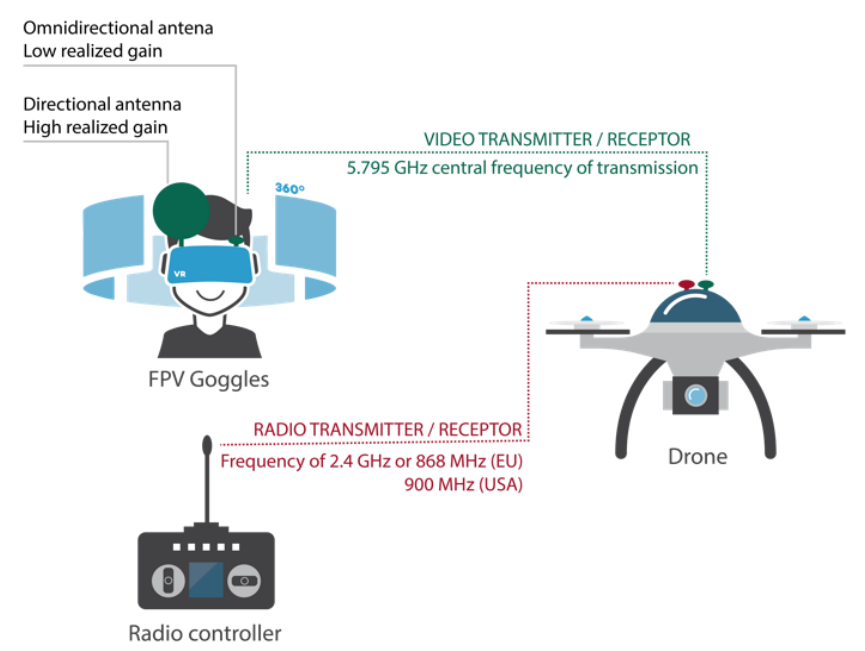

Fig. 1. Complete FPV System.

In practice, this technology requires a connection between a display, which will be the FPV goggles, and the camera onboard of the drone that will transmit the image via a video transmitter. Currently, this connection is made by antennas typically built on a rigid substrate, such as FR-4. The lowcost antennas for FPV can achieve larger sizes, as shown in Fig. 2, and hence significantly increase the weight of the FPV goggles, thus decreasing their wearability. The antenna shown in Fig. 2 is the low-cost Triple Feed Patch Antenna by Maarten Baert [5], and it is widely used in the FPV community. Both the problems mentioned before - the larger size and the added weight, combined with how these antennas connect to the goggles, make them often hang, 
resulting in them being loosely secured. This issue makes them hard to wear, especially if the user is continuously moving or changing his head position.

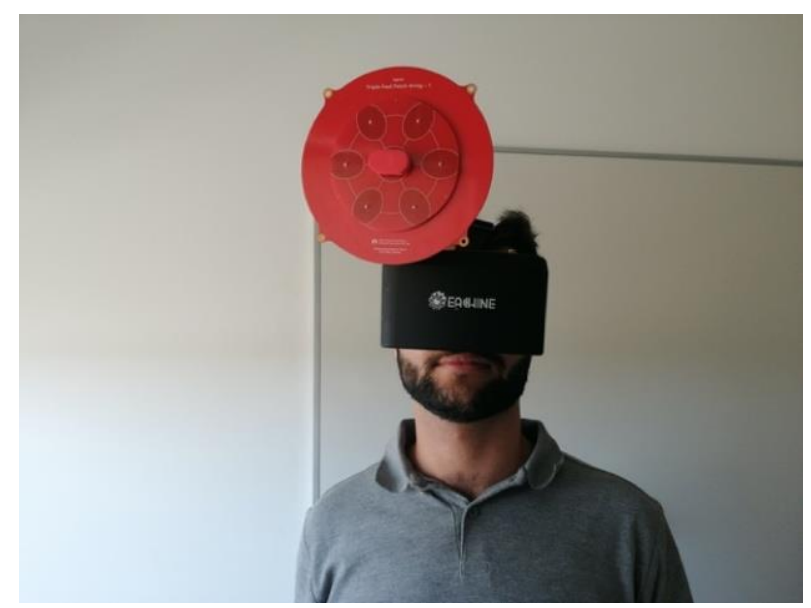

Fig. 2. Example of a rigid antenna currently used in an FPV system [2].

In this paper, an innovative approach is proposed to increase the FPV User Experience (UX). The UX is described as the overall experience of a person using a product, especially in terms of how easy or pleasing it is to use [6]. For this, the use of textile antennas is proposed to reduce the weight of the FPV goggles without reducing the antenna's performance. In this way, it will be possible to increase the comfort of the FPV goggles and to solve the wearability issues.

Firstly, the antenna was designed having in consideration the materials used for its construction. Then, the performance of the designed textile antenna was compared with a commercially available rigid antenna.

This paper is organized as follows. First, a brief overview about textile antennas is given in Section II. The antenna design is presented in Section III. The results of the built antenna are presented in Section IV. Then follows Section V comparing the results of the built antenna with an antenna commercially available for FPV. Conclusions are presented in Section VI.

\section{TeXtile Antennas}

The wearable antenna is the key to make electronic devices less obtrusive. This will enhance the interaction of the user with some electronic devices, making them less invasive and more discrete. Since 2001, when P. Salonen, M. Keskilammi, J. Rantanen, and L. Sydanheimo [7] proposed the first prototype of flexible antenna made of fabrics, the use of textile materials to develop wearable antennas has increased exponentially.

To achieve good results, wearable antennas have to be thin, lightweight, low maintenance, robust, low-cost, and easily integrated in radio frequency circuits [8]. In this way, planar antennas have been proposed for textile applications because they fulfil all these requirements and are adaptable to any surface [9].

To develop this type of antenna, the knowledge of the properties of textile materials is crucial, as well as the knowledge of the manufacturing techniques. Several properties of the materials influence the behavior of the antenna [10]-[11]. Thus, the use of textiles in wearable antennas requires the characterization of their properties. Specific electrically conductive textiles are available on the market and have been successfully used, and ordinary textile fabrics have been used as substrates.

Beyond choosing the textile materials, the construction technique of the antenna is also crucial because the textile materials are highly deformable. The geometrical dimensions of the conductive patch and of the dielectric substrate should remain stable when connecting them, as the mechanical stabilization of both materials is essential to preserve the desired characteristics of the antenna [8]. Several techniques have been explored to manufacture textile antennas, such as laminating [11]-[13], sewing [14], embroidering [15]-[16], and screen printing [17]-[18].

Although textile antennas are used in several applications, no studies were found regarding the FPV ones. Even in the context of rigid antennas for FPV, the only studies found are regarding the ground station's antennas, for instance [19]. In this way, this paper proposes a new application for wearable antennas by designing and testing a textile array for FPV goggles.

\section{ANTENNA DESIGN}

\section{A. FPV Standards}

FPV system antennas must follow a few standards, according to [4]. The frequencies used for FPV transmission are within a range from $5.645 \mathrm{GHz}$ to $5.945 \mathrm{GHz}$, where $5.795 \mathrm{GHz}$ is the central frequency. Therefore, the designed antenna should cover all bandwidth [5]. This paper will focus on designing the directional antenna presented in the FPV system (see Fig. 1). Therefore, to achieve the desired directivity, a $2 \times 2$ antenna array will be used. The realized gain of this type of array is expected to be between $9 \mathrm{dBi}$ and $12 \mathrm{dBi}$. The antenna will also have circular polarization, as it is the most common polarization in FPV systems. The transmitting antenna is located on the drone, which is constantly moving in relation to the reception antenna located on the FPV goggles. In this sense, circularly polarized antennas can increase the received signal energy under these conditions. The polarization chosen for the designed antenna was Right Hand Circular Polarization (RHCP).

\section{B. Design Considerations}

As mentioned in Section I, currently, the commercially available antennas for FPV video reception are made of rigid materials. With the advancement in FPV technology, one of the biggest trends is the increasing portability of the whole system. However, this fact is not observed in the antennas used for video reception. To increase the UX, textile materials were chosen for the antenna construction. Compared with the commercially available antennas, the textile material allows the designed antenna to have a smaller size and a more comfortable utilization since the textile is lighter and more flexible material than the rigid ones.

To design the textile antenna array, commercially available materials were selected and characterized. For the dielectric substrate, the PDE Black (Borgstena Textile Portugal) was chosen. It is a weft-knit fabric with $0.75 \mathrm{~mm}$ of thickness, $\epsilon_{\mathrm{r}}=1.385$, and the loss tangent is of 0.008 . The 
material's dielectric properties were characterized at $5.8 \mathrm{GHz}$, using a resonant-based technique [10]. For the conductive parts, patch and ground plane, the Pure Copper Polyester Taffeta Fabric was used. This conductive material is a $100 \%$ polyester fabric coated with copper and has a thickness of $0.08 \mathrm{~mm}$ and a conductivity of $62500 \mathrm{~S} / \mathrm{m}$.

\section{Single Element Radiation}

To design the full textile array, the first step was to simulate and build a single radiating element. The simulation was made using CST Studio Suite Software. To obtain circular polarization, the upper right and lower left corners were cut out. To achieve the desired resonance frequency and the best axial ratio possible, two parameters were varied, namely, the length of the patch $L$, being this the same as its width and the chamfer length $e$. To check the input impedance and the axial ratio, a line with half wavelength of length was used, as shown in Fig. 3.

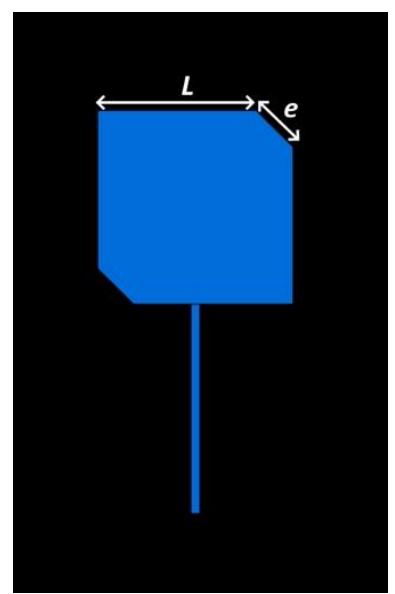

Fig. 3. Single patch design.

\section{Full Array Simulation}

To design the array feeding network, the input impedance of the single radiating elements has to be taken into consideration to assure proper impedance matching. The dimensioning of the feeding network was specially using the smallest number possible of quarter wavelength transformers since they lead to a reduced bandwidth. Furthermore, the usage of thinner lines helps to reduce the radiation effects of the lines. However, it is also important to keep in mind the construction limits. The upper part of the array feeding network consists of a line that connects both radiating patches horizontally. This line has an impedance approximate to the input impedance of the patch, which is approximately $70 \Omega$, and it is defined as L1 and L2 lines in Fig. 4. The length of this line defines the spacing between the radiating elements, which in this case is $3 / 4 \lambda$. This spacing allows a balanced trade-off between antenna parameters, such as gain, half power bandwidth (HPBW), and side-lobe level. The upper part is also connected to a quarter-wavelength transformer identified as L3, with the necessary width to allow the impedance to be transformed from $35 \Omega$ to the desired $100 \Omega$, which is the impedance of the line L4. This line connects the lower part of the array, which is similar to the upper part. Finally, both the upper and lower parts connect to a $50 \Omega$ impedance line L5 where an SMA connector was added. The final size of the array had to be adjusted due to the size limitations of the goggles used. Therefore, the array substrate and ground plane had a size of $80 \mathrm{~mm} \times 80 \mathrm{~mm}$ to fit in front of the goggles. The final array dimensions are shown in Table I, where $l$ is the length and $w$ is the width of the line.

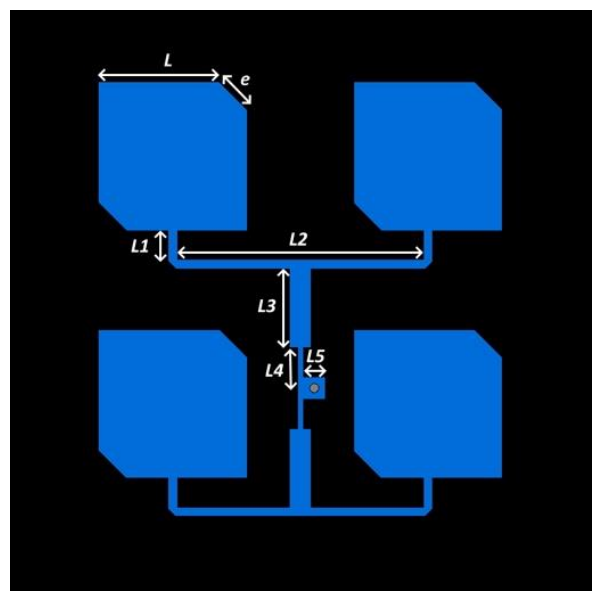

Fig. 4. Full array design.

TABLE I. DIMENSIONS OF THE DESIGNED ANTENNA (UNITS:

\begin{tabular}{|c|c|c|c|c|c|}
\hline \multirow{2}{*}{$\boldsymbol{L}$} & \multirow{2}{*}{$\boldsymbol{e}$} & \multicolumn{2}{|c|}{$\boldsymbol{L 1}$} & \multicolumn{2}{c|}{$\boldsymbol{L 2}$} \\
\cline { 3 - 7 } & & $\mathbf{l}$ & $\mathbf{w}$ & $\mathbf{l}$ & $\mathbf{w}$ \\
\hline 16.89 & 5.44 & 4.71 & 1.31 & 35.60 & 1.31 \\
\hline \multicolumn{2}{|c|}{$\boldsymbol{L 3}$} & \multicolumn{2}{|c|}{$\boldsymbol{4}$} & \multicolumn{2}{|c|}{$\mathbf{5}$} \\
\hline $\mathbf{l}$ & $\mathbf{w}$ & $\mathbf{l}$ & $\mathbf{w}$ & $\mathbf{l}$ & $\mathbf{w}$ \\
\hline 11.00 & 3.02 & 5.64 & 0.88 & 3.10 & 3.00 \\
\hline
\end{tabular}

\section{ANTENNA RESULTS}

To test the performance of the designed array, the textile antenna was manufactured and measured. The textile antenna array was manufactured through the laminating manufacturing technique, where the conductive and dielectric layers of textiles are bonded together using a thermally activated adhesive sheet. In this case, a $100 \%$ polyamide continuous web was used. Furthermore, a Kapton tape was used to stabilize the radiating part of the array, ensuring that none of the lines would deform or move out of place during the laminating process. To ensure the geometric dimensions of the antenna, both textiles (conductive and dielectric ones) were cut using a laser cut machine. Figure 5 shows the manufactured array.

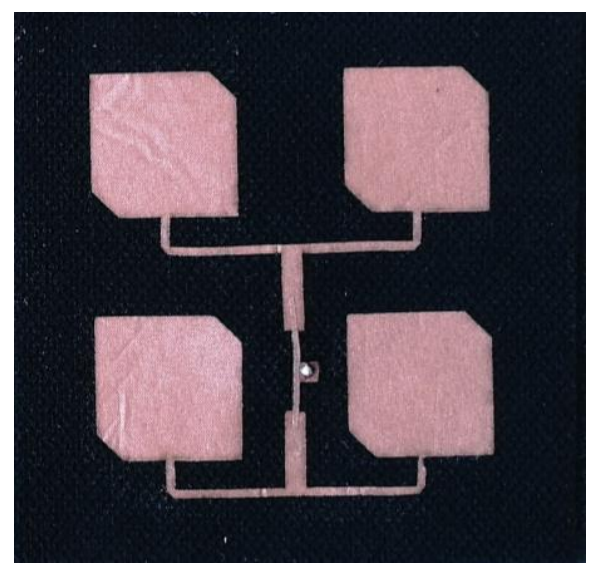

Fig. 5. Manufactured textile array.

After the construction, the performance of the antenna was analysed. The $S_{11}$ parameter was measured in a 
laboratory environment, using a Vector Network Analyzer (VNA) from Anritsu model MS4647B shown in Fig. 6.

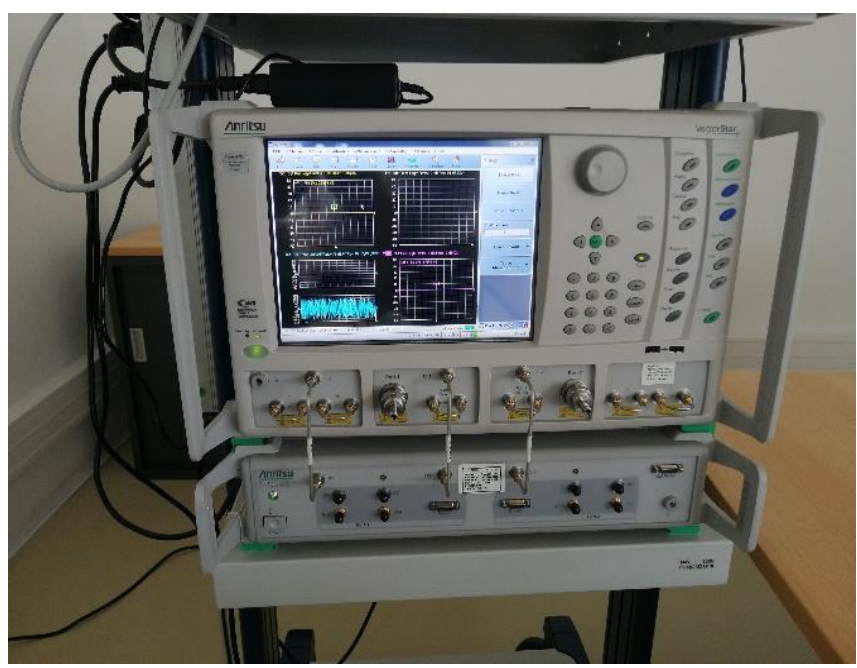

Fig. 6. VNA Anritsu MS4647B used for measurements.

The radiation pattern was measured in anechoic chamber provided by DETI - Departamento de Eletronica,
Telecomunicações e Informática from Aveiro University. Figs. 7-9 present the measured results.

In Fig. 7, it is possible to observe the measured and simulated results of the $S_{11}$ parameter. The simulated results show that the $S_{11}$ is lower than $-10 \mathrm{~dB}$ from $5.473 \mathrm{GHz}$ to $6.260 \mathrm{GHz}$, and it is equal to $-38.91 \mathrm{~dB}$ at the central frequency. The manufactured antenna presented a significant increase in the bandwidth, which it is now between $5.392 \mathrm{GHz}$ and $6.545 \mathrm{GHz}$, resulting in a bandwidth of $1.153 \mathrm{GHz}$. The $S_{11}$ at the frequency of interest $(5.795 \mathrm{GHz})$ was equal to $-17.48 \mathrm{~dB}$.

The antenna gain was also measured being equal to $6 \mathrm{dBi}$, while the simulated one was equal to $9.04 \mathrm{dBi}$. This lower value can be due to the antenna manufacturing process, as it is handmade or also due to high losses inherent to the textile materials. Figure 8 shows the normalized radiation diagram at $\phi=0$ plane of both the manufactured and the simulated antenna. It is possible to extract the half-power beam width (HPBW) that for the simulated antenna was $36^{\circ}$ and the manufactured antenna was approximately $40^{\circ}$. Regarding the axial ratio, the simulated one was equal to $0.32 \mathrm{~dB}$ at the central frequency.

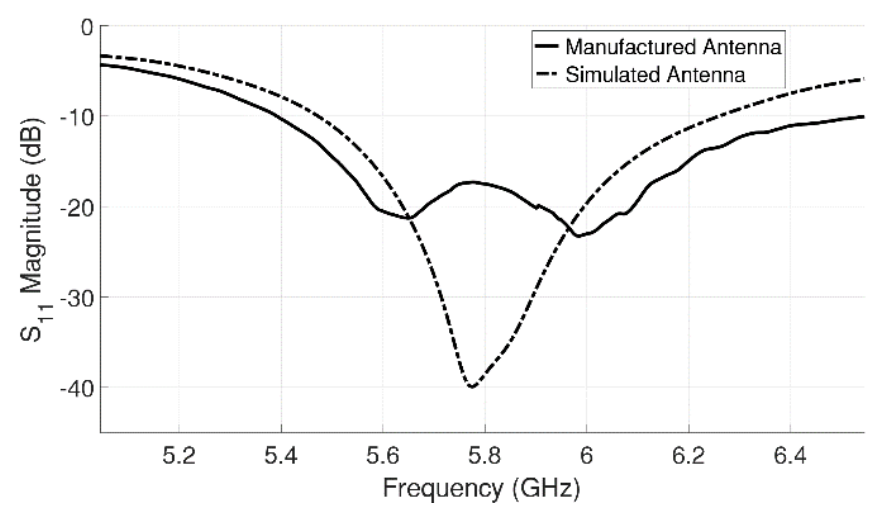

Fig. 7. Comparison of the simulated and measured $S_{11}$ parameter of the textile antenna array.

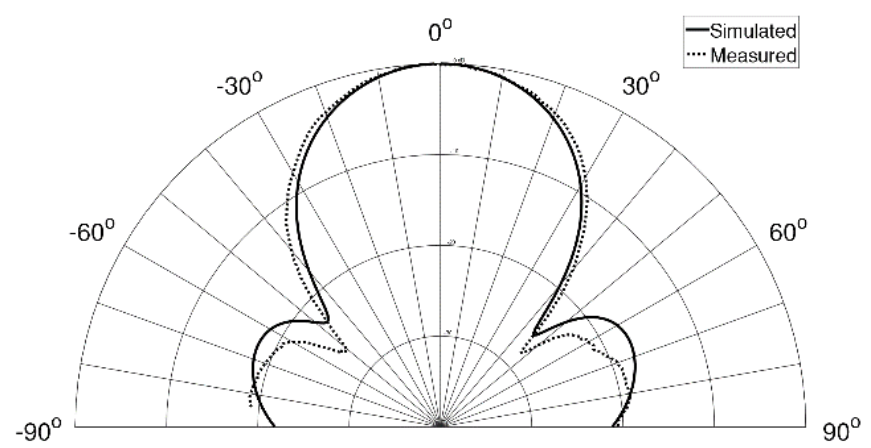

Fig. 8. Comparison of the simulated and measured normalized radiation pattern of the textile antenna array.

\section{COMPARISON WITH A COMMERCIAL ANTENNA}

This section aims to compare the designed textile antenna with an antenna that is widely used by the FPV Drone community. The antenna used in this comparison is the Triple Feed Patch Antenna by Maarten Baert (see Fig. 2), which is made with the FR-4 substrate. Figure 9 presents the comparison of the measured $\mathrm{S}_{11}$ parameters between the designed antenna and the commercially available one.

In Fig. 9, it is possible to observe that both antennas are adapted for the desired frequencies. The commercial antenna has a $\mathrm{S}_{11}$ lower than $-10 \mathrm{~dB}$ from $5.214 \mathrm{GHz}$ to $6.302 \mathrm{GHz}$, resulting in a bandwidth of $1.088 \mathrm{GHz}$, and at the centre frequency, has a value equal to $-15.76 \mathrm{~dB}$. When comparing both antennas, it is possible to observe that the designed textile antenna has a better bandwidth and lower $\mathrm{S}_{11}$ value at the central frequency.

Table II shows the key parameters of both antennas. As shown, the proposed textile antenna has competitive results in almost all aspects. The only result that differs between 
them is the gain, where the designed antenna is slightly lower than the commercial. This is an expected result since the textiles are very lossy materials due to their high porosity.

\begin{tabular}{|c|c|c|}
\hline & Commercial Antenna & Designed Antenna \\
\hline Central Frequency & $5.8 \mathrm{GHz}$ & $5.795 \mathrm{GHz}$ \\
\hline Bandwidth & $1.088 \mathrm{GHz}$ & $1.153 \mathrm{GHz}$ \\
\hline$S_{11}(*)$ & $-15.76 \mathrm{~dB}$ & $-17.46 \mathrm{~dB}$ \\
\hline Antenna Gain & $9.3 \mathrm{dBi}(* *)$ & $6.0 \mathrm{dBi}$ \\
\hline HPBW & $55^{\circ}(* *)$ & $40^{\circ}$ \\
\hline
\end{tabular}

Note: (*) At the central frequency; (**) These values were taken from the official page of the antenna, as these were not measured [3].
Furthermore, both antennas were compared in terms of physical properties. Comparing both antennas side by side, as seen in Fig. 10, the designed antenna has a much smaller footprint than the commercial one. In fact, the designed antenna has $80 \mathrm{~mm} \times 80 \mathrm{~mm}$ against the $120 \mathrm{~mm}$ of diameter of the commercial one. In addition, the designed textile antenna is $17 \mathrm{~g}$ lighter. Furthermore, the way it is placed in the goggles improves the user's mobility and comfort, which is unattainable with the currently available antennas. The combination of all these factors creates a highly competitive antenna capable of competing with the antennas currently available for FPV systems.

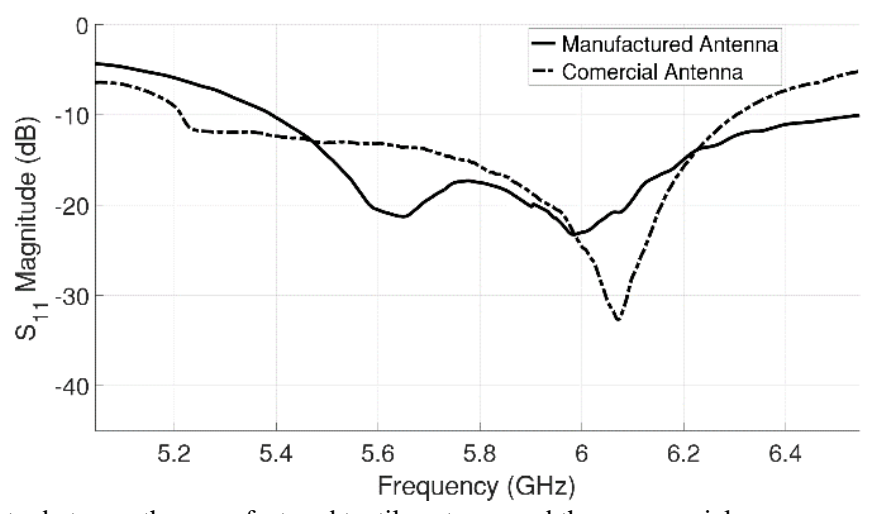

Fig. 9. Comparison of the $S_{11}$ parameter between the manufactured textile antenna and the commercial one.

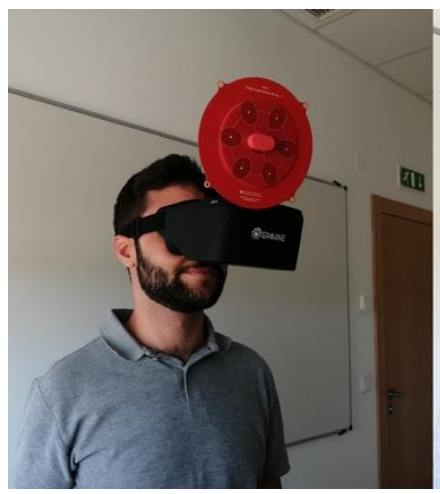

(a)

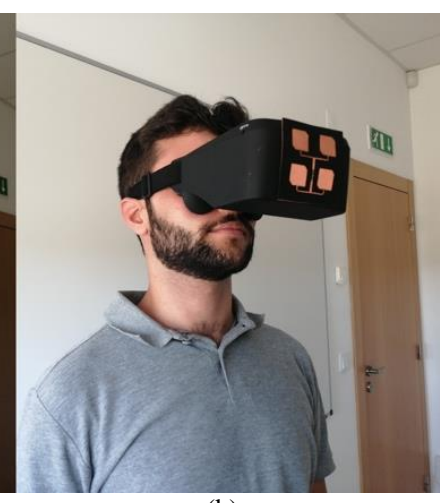

(b)
Fig. 10. Physical comparison between (a) commercial and (b) manufactured antennas.

\section{CONCLUSIONS}

In this work, a solution to the current problems with portability and comfort of FPV antennas was presented. The solution was to use textile antennas instead of the typical rigid substrate. The designed antenna was compared with a commercially available one. This comparison shows promising results for the designed antenna and achieving portability and comfort that cannot be achieved by the antennas currently available while maintaining a gain of $6 \mathrm{dBi}$ and a bandwidth of $1.153 \mathrm{GHz}$ centred at $5.795 \mathrm{GHz}$. The antenna directivity was also maintained achieving a value of HPBW of $40^{\circ}$. The footprint of the designed antenna was also reduced having the designed antenna size of $80 \mathrm{~mm} \times 80 \mathrm{~mm}$. In future work, we intend to explore different types of textile materials, more mechanically stable, allowing for better preservation of the antenna's characteristics and the development of a goggles cover prototype allowing the antenna to be covered and discreet.

\section{CONFLICTS OF INTEREST}

The authors declare that they have no conflicts of interest.

\section{REFERENCES}

[1] A. Saha, A. Kumar, and A. K. Sahu, "FPV drone with GPS used for surveillance in remote areas", in Proc. of $3^{\text {rd }}$ International Conference on Research in Computational Intelligence and Communication Networks (ICRCICN), Kolkata, India, 2017, pp. 62-67. DOI: 10.1109/ICRCICN.2017.8234482.

[2] V. Puri, A. Nayyar, and L. Raja, "Agriculture drones: A modern breakthrough in precision agriculture", Journal of Statistics and Management Systems, vol. 20, no. 4, pp. 507-518, 2017. DOI: 10.1080/09720510.2017.1395171.

[3] U. Stankov, J. Kennell, A. M. Morrison, and M. D. Vujičić, "The view from above: The relevance of shared aerial drone videos for destination marketing", Journal of Travel \& Tourism Marketing, vol. 36, no. 7, pp. 808-822, 2019. DOI: 10.1080/10548408.2019.1575787.

[4] J. Delmerico, T. Cieslewski, H. Rebecq, M. Faessler, and D. Scaramuzza, "Are we ready for autonomous drone racing? The UZHFPV drone racing dataset", in Proc. of International Conference on Robotics and Automation (ICRA), Montreal, 2019, pp. 6713-6719. DOI: 10.1109/ICRA.2019.8793887.

[5] “Triple Feed Patch antenna - Quadcopters - Maarten Baert's website", Maartenbaert.be, 2021. [Online]. Available: https://www.maartenbaert.be/quadcopters/antennas/triple-feed-patchantenna/

[6] R. Polacek, "User interface concept for smart glasses", BSc. thesis, University of Economics in Prague, May 2020.

[7] P. Salonen, M. Keskilammi, J. Rantanen, and L. Sydanheimo, "A novel bluetooth antenna on flexible substrate for smart clothing", in Proc. of IEEE Int. Conf. Syst. Man, Cybern., Tucson, AZ, USA, 2001, pp. 789-794, vol. 2. DOI: 10.1109/ICSMC.2001.973011.

[8] R. Salvado, C. Loss, R. Goncalves, and P. Pinho, "Textile materials for the design of wearable antennas: A survey", Sensors, vol. 12, no. 11, pp. 15841-15857, 2012. DOI: 10.3390/s121115841.

[9] C. A. Balanis, Antenna Theory: Analysis and Design, 3rd ed., Wiley Interscience: Hoboken, NJ, USA, 2005

[10] C. Loss, R. Gonçalves, P. Pinho, and R. Salvado, "Influence of some structural parameters on the dielectric behaviour of materials for 
textile antennas", Textile Research Journal, vol. 89, no. 7, pp. 11311143, 2018. DOI: $10.1177 / 0040517518764000$.

[11] C. Loss, R. Salvado, R. Gonçalves, and P. Pinho, "Influence of the laminating manufacturing technique on the Sn parameter of printed textile antennas", in Proc. of 2017 IEEE MTT-S International Microwave Workshop Series on Advanced Materials and Processes for RF and THz Applications (IMWS-AMP), Pavia, Italy, 2017, pp. 13. DOI: 10.1109/IMWS-AMP.2017.8247426.

[12] C. Hertleer, H. Rogier, L. Vallozzi, and L. Van Langenhove, "A textile antenna for off-body communication integrated into protective clothing for firefighters", IEEE Trans. Antennas Propag., vol. 57, no. 4, pp. 919-925, 2009. DOI: 10.1109/TAP.2009.2014574.

[13] C. Loss, R. Gonçalves, C. Lopes, P. Pinho, and R. Salvado, "Smart coat with a fully-embedded textile antenna for IoT applications", Sensors, vol. 16, no. 6, 2016. DOI: 10.3390/s16060938.

[14] P. Salonen, Y. Rahmat-samii, M. Schaffrath, and M. Kivikoski, "Effect of textile materials on wearable antenna performance: A case Study of GPS antenna", in Proc. of IEEE Antennas and Propagation Society Symposium (APS), 2004, pp. 459-462, vol. 1. DOI: 10.1109/APS.2004.1329673.
[15] A. Tsolis, W. G. Whittow, A. A. Alexandridis, and J. C. Vardaxoglou, "Embroidery and related manufacturing techniques for wearable antennas: Challenges and opportunities", Electronics, vol. 3, no. 2, pp. 314-338, 2014. DOI: 10.3390/electronics3020314.

[16] A. Kiourti, C. Lee, and J. L. Volakis, "Fabrication of textile antennas and circuits with $0.1 \mathrm{~mm}$ precision", IEEE Antennas and Wireless Propagation Letters, vol. 15, pp. 151-153, 2016. DOI: 10.1109/LAWP.2015.2435257

[17] M. L. Scarpello, I. Kazani, C. Hertleer, H. Rogier, and D. Vande Ginste, "Stability and efficiency of screen-printed wearable and washable antennas", IEEE Antennas Wirel. Propag. Lett., vol. 11, pp. 838-841, 2012. DOI: 10.1109/LAWP.2012.2207941.

[18] W. G. Whittow et al., "Inkjet-printed microstrip patch antennas realized on textile for wearable applications", IEEE Antennas and Wireless Propagation Letters, vol. 13, pp. 71-74, 2014. DOI: 10.1109/LAWP.2013.2295942

[19] M. Martínez-Vázquez, "Low-cost RHCP array at $5.8 \mathrm{GHz}$ for FPV ground stations", in Proc. of 2013 IEEE Antennas and Propagation Society International Symposium (APSURSI), Orlando, FL, USA, 2013, pp. 2227-2228. DOI: 10.1109/APS.2013.6711772

This article is an open access article distributed under the terms and conditions of the Creative Commons Attribution 4.0 (CC BY 4.0) license (http://creativecommons.org/licenses/by/4.0/). 